\title{
A System Architecture for Energy Saving in Buildings
}

\author{
Zhao Shi ${ }^{1,2}$, Yang Liu ${ }^{1}$, Xing Tong ${ }^{1}$, Peng Zeng ${ }^{1}$, Haibin $\mathrm{Yu}^{1}$ \\ ${ }^{1}$ Department of Industrial Control Network and System \\ Shenyang Institute of Automation Chinese Academy of Sciences \\ Shenyang, Liaoning, 110016, China \\ ${ }^{2}$ University of Chinese Academy of Sciences \\ Beijing, 100049, China \\ E-mail: shizhao@sia.cn
}

\begin{abstract}
Advances in smart grid technologies have been proven to improve energy efficiency. These technologies will drive significant changes of energy consumption operation, energy management, energy using patterns, and customer services. In order to achieve energy saving efficiently and provide smart services automatically for users, the architecture of a smart energy monitoring and management system is proposed in this paper. By monitoring the power consumption information, environment information and users' situation information, the system based on the architecture can calculate the proportion of wasted energy consumption based on the energy consumption statistic, provide smart services based on the person-device interaction, and forecast the energy consumption based on the user energy consumption behaviors. The system is combined with cloud computing for data storage and processing. This paper describes the design and partial implementation of the system architecture.
\end{abstract}

Keywords-smart grid, energy consumption statistic, persondevice interaction, user energy consumption behaviors, cloud computing

\section{INTRODUCTION}

With the rapid development of society after the Industrial Revolution, fossil fuel is being exhausted because of our insatiable thirst for energy. Therefore, energy saving is an important topic of our age.

Researchers have recently focused on smart applications with intelligent sensors, because the majority of the energy consumption is used for human activities. Examples include the simple equipment that intelligently controls the Voiceactivated lights based on the voice and the intensity of illumination in the corridor of our buildings. [1]proposed a power-aware LED light enabler system to control the LED light based on user's situation.

Except for using intelligent sensors, some studies on energy distribution and management system have been done. Some systems implement the building energy saving by changing the user behavior based on the data statistic for devices. [2]proposed a high-fidelity energy monitoring and feedback architecture for reducing energy consumption in buildings through the visualization of energy consumption information to users. But they did not have any intelligent suggestions and policies combining the user's situation and information, and they did not deal with a significant amount of data efficiently. Others make energy consumption efficient and intelligent with user situation information, device information and sensor node management. [3][4]proposed an intelligent system for building energy saving and context-aware smart services. The system achieves energy saving by monitoring the user situation and controlling the electrical devices automatically. But the system only provides energy consumption statistics in aggregate. It is insufficient to identify where the energy is wasted and the proportion of wasted in the energy consumption.

For this reason, we are designing a smart energy monitoring and management system with cloud computing for energy saving and context-aware services in buildings. The main features of our system are as follows:

1) Cloud computing: Cloud computing has been a rising network application mode in the past five years. This application is unique in that it is entirely built on a layer of self-maintenance and management of virtual resources. Users are able to dynamically change the type and quantity of the resources and services which need to be accessed according to the different needs. Meanwhile, cloud computing is able to store and process a significant amount of data efficiently. Existing systems for energy saving and context-aware services do not use cloud computing for data storage and processing. We have used cloud computing for storing and processing all the data in our data storage module.

2) Energy consumption statistic for low voltage devices: The voltage is divided into the high voltage and the low voltage inside the building. The focus of our system is on studying the energy consumption of the low voltage devices such as lighting, LCD, Laptop, desktop and air conditioning, etc. Our system measures the energy consumption of each device with high-frequency and highdensity. Through energy consumption statistic of devices, we can acquire the energy consumption of related users within a certain period of time and calculate the proportion of wasted energy consumption based on the situation of users.

3) Person-device interaction based on context-aware technology: A context-aware technology is aware of the user's state and surroundings and modifies system behavior 
based on this information[5]. Inside a typical commercial office building, users and devices form a corresponding relationship in our system. The status of devices will be changed based on the change of the situation of the related user. The statistics of user's situation and energy consumption are able to infer the energy consumption behavior of the user and the proportion of wasted power consumption.

4) Energy consumption forecasting based on the user behavior: The statistics of user's situation and energy consumption are able to infer the energy consumption behavior of the user. In return, the energy consumption behavior of the user is able to forecast the user's energy consumption. The load forecasting is useful for electricity distribution of the power grid.

\section{RELATED WORKS}

Some recent studies focused on the energy consumption statistic of both devices of the low voltage and the high voltage. [2]proposed a high-fidelity energy monitoring and feedback architecture for reducing electrical consumption. It proposed the load tree for energy data analysis and visualization. Based on the feedback and visualization of the energy consumption to users in the application interface, users change their energy consumption behavior in order to save energy in buildings. And the energy manager in the building is able to know the energy consumption by reaggregating the load tree by different functions.

Other studies focused on situation-awareness in order to effectively provide the building control and energy saving services. [3][4]proposed an intelligent system with some sensors in each domain to aware the user and environment information for building energy saving and context-aware smart services. This system uses the context-aware technology to control electrical devices based on the information of user's situation, environment, and sensor management for building energy saving. Because the system used sensors to monitor the user's movement, the effect of energy saving is dependent on the number of sensors.

Except for these systems, [6]proposed a new infrastructure called Sensor-Cloud infrastructure which manages physical sensors on IT infrastructure. The infrastructure virtualizes physical sensors as a virtual sensor on the cloud computing for managing and provisioning sensors effectively. [7]proposed a methodology of forecasting long term electrical energy demand. [8]proposed a new fuzzy logic method for midterm energy forecasting.

Compared to these existing works, we propose the architecture of a smart energy monitoring and management system for energy saving and context-aware services in buildings combining cloud computing. The system is implemented to measure and analyze energy consumption, achieve person-device interaction based on context-aware technology, and forecast energy consumption based on the user energy consumption behavior.

\section{ARCHITECTURE}

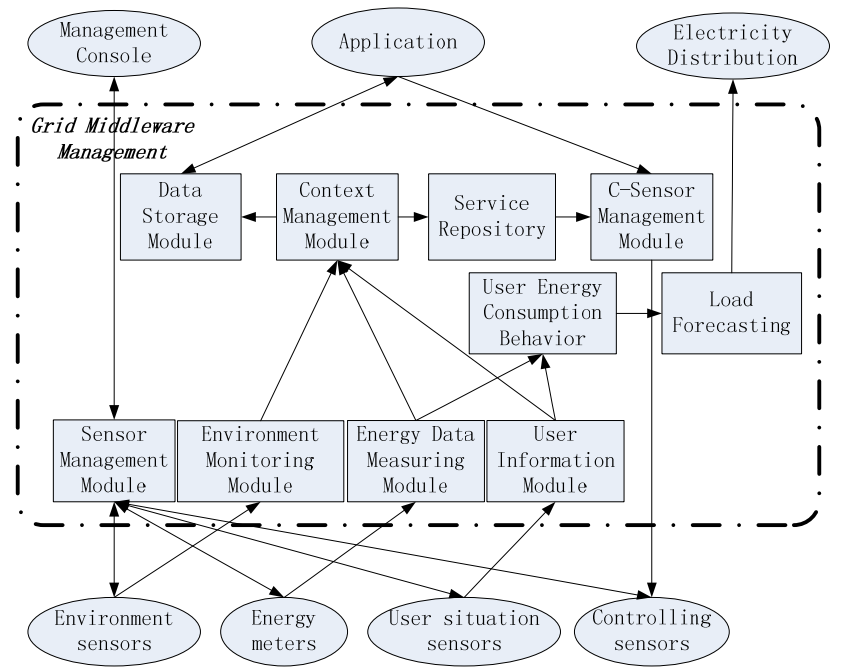

Figure 1. The architecture of our system

In this paper, we present the architecture of our smart energy monitoring and management system with cloud computing for energy saving and context-aware services in buildings. Figure 1 shows the architecture of our system.

The system is composed of three parts: a management and application part, a grid middleware management part, and a sensing and measuring part.

\section{A. Management and application part}

The management and application part is composed of three components: a management console, an application module, and the electricity distribution part of the power grid.

1) Management console: This console is for the administrator to monitor and manage environment sensors which monitor the temperature, humidity and intensity of illumination information, energy meters which measure electrical power, user situation sensors which aware users' situation and controlling sensors which control devices. The administrator also needs to maintain the operation of the whole system.

2) Application module: This module is for users to retrieve the energy consumption information and control electrical devices. It gets the information of the energy consumption from cloud computing. It also performs related control operations to devices through C-Sensor management module.

3) Electricity distribution: The electricity distribution is a part of the power grid, which delivers electricity to end customers. If it forecasts the approximate energy consumption before the energy is distributed in the electricity distribution part of the power grid, electricity generation and distribution will be more planned. It contributes to reduce the fluctuations in electricity consumption, so as to achieve energy saving.

\section{B. Grid middleware management part}

The grid middleware management part is composed of ten components: a sensor management module (SMM), an 
environment monitoring module (EMM), an energy data measuring module (EDMM), a user information module (UIM), a user energy consumption behavior module (UECB), the load forecasting (LF), a data storage module (DSM), a context management module (CMM), the service repository (SR), and a c-sensor management module (CSMM).

1) SMM: This module manages environment sensors, energy meters, user information sensors and controlling sensors. SMM receives sensor controlling commands from the management console and manages the sensors. It also monitors all sensors and notifies the management console if some of them are disconnected.

2) EMM: This module receives the information of environment from the environment sensors and sends it to the context management module.

3) EDMM: This module receives the information of device energy consumption from the energy meters and sends it to the context management module and the user energy consumption behavior module.

4) UIM: This module receives the information of user's situation from the user situation sensors and sends it to the context management module and the user energy consumption behavior module.

5) UECB: This module manages the energy consumption behavior of users. UECB receives the information of energy consumption and user's situation from the energy data measuring module and the user information module respectively. Then it analyzes the information and generates the user behavior for load forecasting.

6) LF: This module sends the energy consumption forecasted to the electricity distribution. It receives the information of user energy consumption behavior, forecasts the energy consumption the user will use, and sends it to the electricity distribution.

7) DSM: This module stores and processes the data which include the environment information, energy data and user information. It also provides the data to the application based on query commands. In this module, our system has used a distributed storage for querying and processing data. There are two databases in our system: the HBase and the Oracle. The Oracle database is used to store the data of the day for real-time displaying function in the application. The HBase database is used to store all the data. Because the HBase has an advantage only in the query and processing of a significant amount of data, we can query and process realtime data efficiently by using the Oracle database.

8) CMM: This module real-time manages the context based on context-aware technology and analyzes the information of environment, energy consumption and user. CMM receives the information from the environment monitoring module, energy data measuring module and user information module. After analysis and processing of the information, CMM sends the processed information to the data storage module and the context analysis results to the service repository to provide services.

9) SR: This module provides the services and operations to the c-sensor management module. SR receives the context results from the context management module, and matches the related services from the repository or creates new services. Then it sends the controlling command to the c-sensor management module.

10) CSMM: This module manages devices through controlling sensors. CSMM receives controlling commands from the application module or the service repository, and sends them to controlling sensors to perform related operations to devices.

\section{Sensing and measuring part}

The sensing and measuring part is composed of four components: environment sensors, energy meters, user situation sensors, and controlling sensors.

1) Environment sensors: This component includes all sensors which monitor the environment information, such as temperature sensors, humidity sensors, and intensity of illumination sensor, etc. These sensors send the information of environment to the environment monitoring module.

2) Energy meters: This component includes all electrical meters which measure the energy consumption. These meters send the information of energy consumption to the energy data measuring module.

3) User situation sensors: This component includes all sensors which monitor the users' movement and situation information. These sensors send users information to the user information module.

4) Controlling sensors: This component includes all sensors which control all electrical devices used by users. These sensors receive operation commands from the csensor management module and then control devices.

The important components in the system architecture are how to optimize the query in the database, how to provide the energy saving policy combining the environment information, user information and energy consumption information in the context management module, and how to record the energy consumption behavior of users and forecast the energy consumption in the user energy consumption behavior module.

\section{IMPLEMENTATION}

We have implemented some parts of our system. We have implemented cloud computing to store the significant amount of data, energy meters to measure the electrical data, controlling sensors to control devices, and the application to show the energy consumption for each device and to control the device. We are arranging user situation sensors to monitor the movement and situation information of users, and environment sensors to monitor the environment information. And we will design the context management module and the user energy consumption behavior module for context-aware smart services and load forecasting respectively.

\section{CONCLUSIONS AND FUTURE WORKS}

Advances in smart grid technology have improved energy efficiency. This paper proposes an architecture of a smart energy monitoring and management system with cloud computing for building energy saving and context-aware 
smart services. We consider integration of cloud computing for storing and analyzing data, energy consumption statistic for low voltage devices, person-device interaction based on context-aware technology, and energy consumption forecasting based on the user behavior in order to make the energy consumption more efficient and intelligent. We have implemented some parts of our system. And there is some work being done and will be done. We expect that our work will contribute to the development of building energy monitoring and management system for building energy saving and context-aware smart services.

\section{ACKNOWLEDGMENT}

The authors acknowledge the financial support of the Strategic Priority Research Program of the Chinese Academy of Sciences under Grant No.XDA06020302, the Important National Science and Technology Specific Project under Contact No.2010ZX03006-005-01, the National High Technology Research and Development Program of China under 863 Program No.2011AA040103, and the Natural Science Foundation of China under Contact No.61100159.

\section{REFERENCES}

[1] Y. Uhm, I. Hong, G. Kim, B. Lee and S. Park, "Design and Implementation of Power-aware LED Light Enabler with Locationaware Adaptive Middleware and Context-aware User Pattern,"
Consumer Electronics, IEEE Transactions on, vol.56, pp.231-239, 2010

[2] X.F. Jiang. "A High-Fidelity Energy Monitoring and Feedback Architecture for Reducing Electrical Consumption in Buildings". California, Berkeley, 2010.

[3] J. Byun and S. Park. "Development of a Self-adapting Intelligent System for Building Energy Saving and Context-aware Smart Services" Consumer Electronics, IEEE Transactions on, Vol.57, pp.90-98, 2011

[4] J. Byun, I. Hong, B. Kang, and Sehyun Park. "A Smart Energy Distribution and Management System for Renewable Energy Distribution and Context-aware Services based on User Patterns and Load Forecasting" Consumer Electronics, IEEE Transactions on, Vol.57, pp.436-444, 2011

[5] A. Krause, A Smailagic and D. P. Siewiorek, "Context-Aware Mobile Computing: Learning Context-Dependent Personal Preferences from a Wearable Sensor Array," Mobile Computing, IEEE Transactions on, vol.5, pp.113-127, 2006.

[6] M. Yuriyama and T. Kushida."Sensor-Cloud Infrastructure”.The 13th International Conference on Network-Based Information Systems, 2010.

[7] Q.Ahsan and M. Uddin. "A Probabilistic Approach of Electrical Energy Forecasting”. Instrumentation and Measurement Technology Conference, 2005.

[8] C. N. Elias and N. D. Hatziargyriou. "An Annual Midterm Energy Forecasting Model Using Fuzzy Logic” Power Systems, IEEE transactions on, Vol.24, pp.469-478, 2009. 